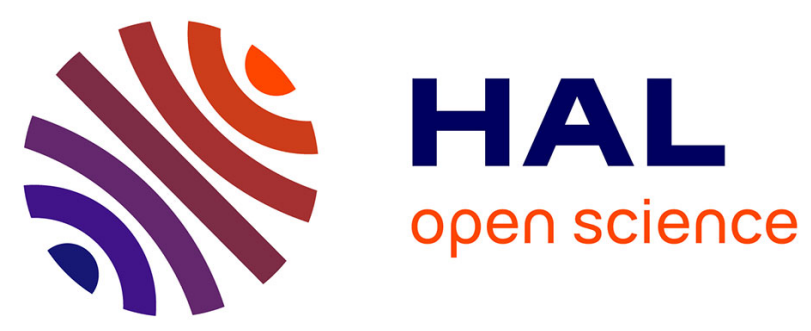

\title{
Nonequilibrium Precondensation of Classical Waves in Two Dimensions Propagating through Atomic Vapors
}

\author{
Neven Šantić, Adrien Fusaro, Sabeur Salem, Josselin Garnier, Antonio
} Picozzi, Robin Kaiser

\section{- To cite this version:}

Neven Šantić, Adrien Fusaro, Sabeur Salem, Josselin Garnier, Antonio Picozzi, et al.. Nonequilibrium Precondensation of Classical Waves in Two Dimensions Propagating through Atomic Vapors. Physical Review Letters, 2018, 120 (5), pp.055301. 10.1103/PhysRevLett.120.055301 . hal-03533023

\section{HAL Id: hal-03533023 \\ https://hal.science/hal-03533023}

Submitted on 11 Feb 2022

HAL is a multi-disciplinary open access archive for the deposit and dissemination of scientific research documents, whether they are published or not. The documents may come from teaching and research institutions in France or abroad, or from public or private research centers.
L'archive ouverte pluridisciplinaire HAL, est destinée au dépôt et à la diffusion de documents scientifiques de niveau recherche, publiés ou non, émanant des établissements d'enseignement et de recherche français ou étrangers, des laboratoires publics ou privés. 


\title{
Non-equilibrium precondensation of classical waves in two dimensions propagating through atomic vapors
}

\author{
Neven Santićc ${ }^{1,2}$, Adrien Fusaro ${ }^{3}$, Sabeur Salem ${ }^{1}$, Josselin Garnier ${ }^{4}$, Antonio Picozzi ${ }^{3}$, Robin Kaiser $^{1}$ \\ ${ }^{1}$ Université Côte d'Azur, CNRS, Institut de Physique de Nice, Valbonne F-06560, France \\ 2 Institute of Physics, Bijenička cesta 46, 10000 Zagreb, Croatia \\ 3 Laboratoire Interdisciplinaire Carnot de Bourgogne, CNRS, \\ Université Bourgogne Franche-Comté, Dijon, France and \\ ${ }^{4}$ Centre de Mathematiques Appliquées, Ecole Polytechnique, 91128 Palaiseau Cedex, France
}

\begin{abstract}
The nonlinear Schrödinger equation, used to describe the dynamics of quantum fluids, is known to be valid not only for massive particles, but also for the propagation of light in a nonlinear medium, predicting condensation of classical waves. Here we report on the initial evolution of random waves with Gaussian statistics using atomic vapors as an efficient two dimensional nonlinear medium. Experimental and theoretical analysis of near field images reveal a phenomenon of nonequilibrium precondensation, characterized by a fast relaxation towards a precondensate fraction of up to $75 \%$. Such precondensation is in contrast to complete thermalization to the Rayleigh-Jeans equilibrium distribution, requiring prohibitive long interaction lengths.
\end{abstract}

Bose-Einstein condensation has been reported in a variety of quantum systems, such as ultracold atoms and molecules [1], exciton polaritons [2-4], or photons [5], where the bosonic character of the particles is crucial. On the other hand, it is known that an ensemble of classical waves can exhibit a phenomenon of condensation, whose thermodynamic properties are analogous to those of the genuine quantum Bose-Einstein condensation, despite the classical nature of the system [6-15]. Indeed for waves traveling in random directions in a nonlinear medium, wave thermalization and condensation can occur. Such spontaneous formation of large scale coherent structures is encountered in many fields of physics, such as astrophysics, low-temperature condensed matter, hydrodynamics, plasma physics and optics. Contrary to dissipative systems, conservative Hamiltonian systems cannot evolve towards a fully ordered state, because such an evolution would imply a loss of statistical information for the system, which would violate its formal reversibility. However, in spite of its formal reversibility, a nonintegrable Hamiltonian system can exhibit a self-organization process which is induced by its natural thermalization towards the equilibrium state $[6-10,12,14-20]$. Wave condensation is a spectacular example of this type of selforganization processes, which results from the divergence of the classical Rayleigh-Jeans equilibrium distribution.

Here we present an experimental system allowing us to study the time evolution of such wave condensation in two dimensions. In contrast to ultracold atom experiments, the wave under consideration is the electromagnetic field of a laser beam, rendered spatially incoherent by passing through a diffuser. At variance with many ultracold atom experiments, we also consider here a situation without an external potential.

An important aspect of this experimental work is the study of fast relaxation to out of equilibrium states in the initial process of two-dimensional thermalization. Indeed achieving complete thermalization and condensa-

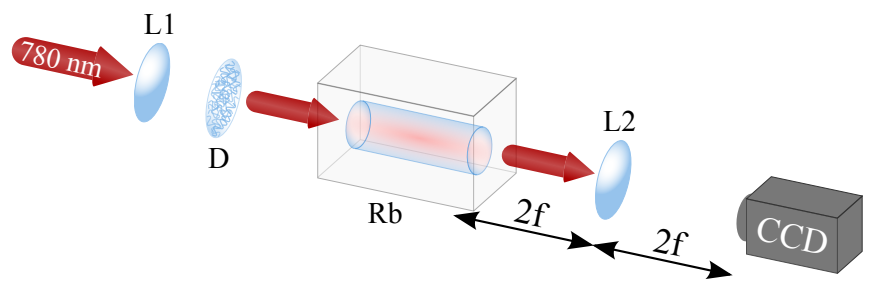

FIG. 1: Experimental setup. After passing a first lens $(L 1)$ a $1 W$ laser is impinging on a diffuser $(D)$ creating a speckle field at the input face of a heated atomic vapor cell containing rubidium atoms. The output field is imaged on a CCD camera using a second lens ( $L 2)$ with focal distance $f$.

tion of random nonlinear waves through nonlinear optical propagation is known to require prohibitive large interaction lengths [17-19]. The existence of fast relaxation to out of equilibrium states is an open problem that is attracting a growing interest in different research communities [21-24], including long range interacting systems, where fast relaxation occurs towards quasi-stationary states $[25,26]$ or one dimensional nearly integrable quantum systems, where experimental signatures of prethermalization have been observed [27-29]. At variance with the usual approach characterizing wave condensation in the far field spectrum, here, we identify a fast initial relaxation through the analysis of the optical near field, which reveals the existence of a phenomenon of precondensation that occurs far from thermal equilibrium for short propagation lengths. Our work thus contributes an experimental observation (supported by numerical simulations) of fast relaxation to out of equilibrium states.

The nonlinear Schrödinger equation (NLS) describing the experiment can be rewritten as [30]:

$$
i \frac{\partial \psi}{\partial z}=-\frac{1}{2 k_{0}} \nabla^{2} \psi+\gamma|\psi|^{2} \psi
$$


where $\nabla$ is the gradient in the transverse surface section of the beam $\boldsymbol{r}=(x, y)$, while the longitudinal variable $z$ plays the role of the time evolution. The wavelength of the optical wave is $\lambda=2 \pi / k_{0}$ and $\gamma$ describes the strength of the nonlinearity. The incident speckle field is characterized by a transverse correlation length $\sigma_{c}$, which also determines the initial transverse momentum distribution. The healing length denotes the relevant transverse length scale for which linear and nonlinear effects are of the same order $\Lambda=\sqrt{z_{N L} /\left(2 k_{0}\right)}$, where $z_{N L}=$ $1 /\left(\gamma I_{0}\right)$ is the nonlinear length scale, and $I_{0}=\left\langle|\psi|^{2}\right\rangle$ is the intensity averaged over the relevant transverse surface of the beam (see below). In this work we consider defocusing nonlinearities $(\gamma>0)$, corresponding to repulsive interactions. We recall that, in addition to the intensity $I_{0}$ ('particle number'), the NLS Eq.(1) also conserves the total energy (Hamiltonian) $H=E+U$, which has a kinetic (linear) contribution $E(z)=\frac{1}{2 k_{0}} \int|\nabla \psi|^{2} d \boldsymbol{r}$, and a nonlinear contribution $U(z)=\frac{\gamma}{2} \int|\psi|^{4} d \boldsymbol{r}$.

We stress that this type of classical wave condensation occurs in the spatial frequency domain and at the same wavelength as that of the incident laser. This is in contrast to the condensation of photons reported in [5], where the effect of condensation also occurs for temporal frequencies and is accompanied with inelastic light scattering via interactions with a thermal bath. In the situation considered here, no thermal bath is present: we deal with a microcanonical statistical description, where the total energy $H$ plays a role analogous to the temperature (note that, in analogy with kinetic gas theory, the kinetic energy $E(z)$ provides a natural measure of the amount of randomness in the incoherent wave). This is a key difference with respect to the broader notion of condensation used to characterize different phenomena in optical cavities, which are inherently forced-dissipative systems [4, 42-46]. Also, at variance with quenched cooling achieved in ultracold atom experiments [47-50], here the initial condition explored does not include any truncation of higher transverse momenta. The possibility to engineer the initial conditions for the nonlinear propagation illustrates the potential of this experiment to explore novel regimes of the universal two dimensional NLS equations.

The experimental setup is depicted in Fig. 1. The output of a $1 \mathrm{~W}$ fibre laser, tuned below the D2 line of Rb at $780 \mathrm{~nm}$ (allowing for defocusing nonlinearities [30]), is used to realize a speckle field by passing through a diffuser providing a Gaussian distribution of incident wavevectors in the transverse plane. The correlation length of the speckle field can be adjusted by changing the size of the illumination area on the diffuser (see Fig. 1). This allows us to tune the kinetic contribution $E(z=0)$. This speckle field is then sent onto the atomic vapor with a Gaussian envelope on the order of $w_{s p} \approx 5 \mathrm{~mm}$ and the average intensity $I_{0}$ around the center of the beam is thus proportional to the total power $I_{0} \propto P / w_{s p}^{2}$. The
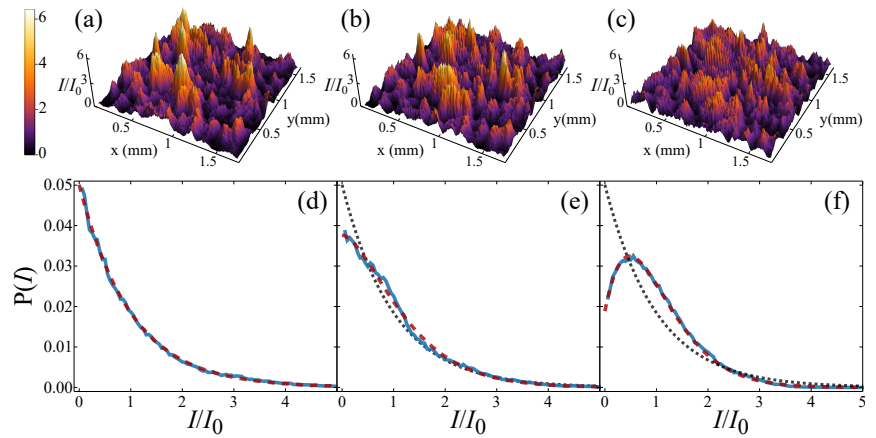

(d)
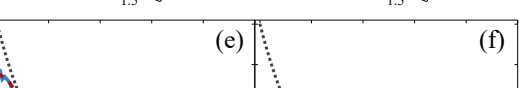

(f)

FIG. 2: Near Field Speckle: (a)-(c) near field images for $L / z_{N L}=0,3.5 \pi$ and $14.4 \pi$; (d)-(f): corresponding intensity histograms, showing the emergence of a non zero value for the maximum of $P(I)$ with corresponding precondensation fractions $n_{0}$ : (d) $n_{0}=0$, (e) $n_{0} / I_{0}=0.5$, (f) $n_{0} / I_{0}=0.7$. The dotted black line refers to the exponential (Gaussian statistics), the dashed red line is a fit to the probability density (2).

nonlinear medium consists of a $L=7 \mathrm{~cm}$ long heated vapor cell containing a natural mixture of rubidium atoms. By adjusting the temperature of the cell, we can vary the atomic density $\rho_{a t}$ of the atoms by several orders of magnitude. The narrow atomic resonances allow efficient control of the linear index of refraction and its nonlinearity is due to the excited state saturation, which can be tuned by changing the incident laser frequency $\omega_{L}$ away from the atomic resonance $\omega_{a t}$ by $\Delta=\omega_{L}-\omega_{a t}$ or by adapting the power of the incident laser beam. We are thus able to realize a nonlinear phase shift up to $\Phi_{N L}=k_{0} L \Delta n=20 \pi$, with a nonlinear index of refraction of $\Delta n=20 \pi /\left(k_{0} L\right) \approx 10^{-4}$ and a transmitted power larger than $70 \%$, so that $z_{N L}=L / 20 \pi \approx 1 \mathrm{~mm}$ and $\Lambda \approx 10 \mu \mathrm{m}[30]$.

The light transmitted after nonlinear propagation can be analyzed either in real space (near field) through imaging onto a CCD camera (see Fig. 1), or by investigating the (far field) momentum distribution. Experimental measurements of the far field spectrum are delicate and extremely sensitive to details of the optical setup and detection scheme [51]. At variance with [14], our analysis is based on near field measurements, which will be shown to provide the appropriate framework to define the notion of 'non-equilibrium precondensation'.

In Fig. 2 we illustrate the experimental results of the near field data for increasing values of $L / z_{N L}$, obtained by changing the nonlinear distance $z_{N L}$ at constant $L$. As the laser intensity in the wings of the Gaussian envelope vanishes, the nonlinear interaction is prominent only in the central part of the speckle field. We therefore analyzed the near field intensity by performing spatial averaging $(\langle\rangle$.$) within a sufficiently small region of inter-$ est of the beam where the statistics of the random wave is almost homogeneous. 
For a very far detuned laser inducing a vanishing nonlinearity the transmitted intensity distribution $P(I)$ exhibits an exponential decrease (see Fig. 2(d)), as expected for random waves with Gaussian statistics. By increasing the nonlinearity, the probability distribution strongly deviates from Gaussianity and the maximum of $P(I)$ gradually shifts away from $I=0$ (see Fig. 2(f)). We remark that such a deformation of the distribution is a robust phenomenon that also occurs for non-Gaussian heavy tailed statistics. Also note that apparently similar deformations of the probability density have been reported in 1D optical systems [52-54]. However, the 1D NLS equation is integrable and does not exhibit thermalization or precondensation, so that the $1 \mathrm{D}$ probability density $P(I)$ is of different nature than in 2D [30]. Notice that, owing to an exact relation based on energy conservation, a deviation from Gaussian statistics can be related to the variation of the spectral width of the wave [55].

In analogy with Bose-Einstein condensation, the deformation of the probability distribution in Fig. 2 reflects a reduction of intensity fluctuations that precedes the establishment of long range phase coherence $[56,57]$. We analyze the transmitted near field distribution by decomposing the field into a homogeneous (plane-wave) condensate component and an incoherent component with statistical Gaussian fluctuations, $\psi(\boldsymbol{r}, z)=\sqrt{n_{0}}+\phi(\boldsymbol{r}, z)$. Although the coherent component does not refer to a purely homogeneous plane-wave, such a decomposition proves robust and relevant to our analysis [30]. The intensity distribution $P(I)$ is given then by:

$$
P(I)=\frac{\exp \left(-\frac{I+n_{0}}{I_{0}-n_{0}}\right)}{I_{0}-n_{0}} \mathcal{I}_{0}\left(\frac{2 \sqrt{n_{0} I}}{I_{0}-n_{0}}\right),
$$

where $\mathcal{I}_{0}(x)$ is the modified Bessel function of zero-th order, and $I_{0}=\langle I\rangle$. In the limit $n_{0} \rightarrow 0$, the distribution (2) (also known as Rice-Nakagami distribution) reduces to a pure exponential which characterizes a Gaussian field, $P(I)=\exp \left(-I / I_{0}\right) / I_{0}$. In the opposite limit, $n_{0} / I_{0} \rightarrow 1$, one obtains $P(I)=\delta\left(I-I_{0}\right)$ as expected for a pure condensate plane-wave solution. It is interesting to note that, according to (2), the precondensate is simply related to the variance of the intensity fluctuations, $n_{0} / I_{0}=\sqrt{2-\left\langle I^{2}\right\rangle / I_{0}^{2}}$, which, by energy conservation, is related to the prethermalized kinetic energy. Note in Fig. 2 that the intensity distributions observed in our experiment remain broad. This invalidates the standard Bogoliubov approach $[1,23,58]$, which requires a sharp peaked intensity histogram around $I_{0}$.

We stress the fact that Eq. (2) does not require the field to be in a thermal equilibrium state, so that the probability density (2) is valid even far from equilibrium. Note that this is in contrast with the equilibrium probability density that is derived on the basis of equilibrium statistical mechanics, see Ref.[53]. Actually, the precondensation effect occurs very far from thermal equilibrium

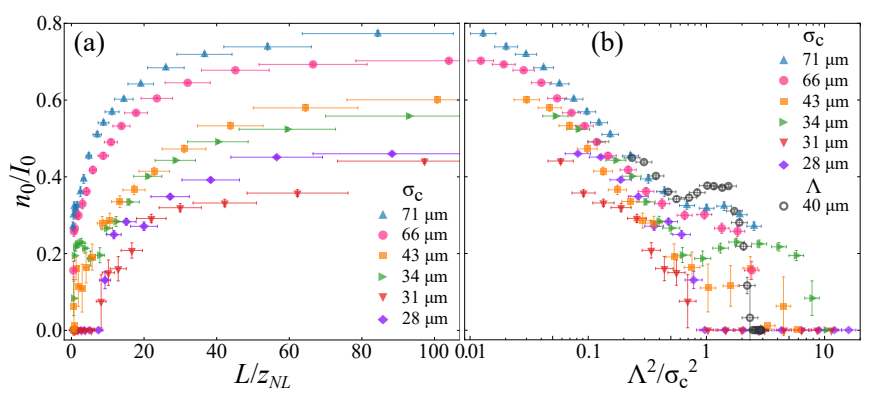

FIG. 3: Precondensate fraction at the constant laser power $(P=1 W)$ and sample length $(L=7 \mathrm{~cm})$ : (a) as a function of propagation distance $L / z_{N L}$ for different values of the speckle correlation length $\sigma_{c}$. The nonlinear length $z_{N L}$ is varied via the laser frequency $\Delta$. (b) as a function of $\Lambda / \sigma_{c}$, obtained by changing the healing length $\Lambda$ via the laser detuning for various correlation lengths $\sigma_{c}$ (full symbols) or by changing the correlation length $\sigma_{c}$ at constant detuning and corresponding healing length $\Lambda=44 \mu m$ (gray circles). Error bars are derived from uncertainties of the nonlinear phase calibration and from fitting respectively.

and does not require the establishment of an equilibrium state, i.e., the Rayleigh-Jeans spectrum. This is a striking difference with the usual equilibrium condensation arising from the divergence of the Rayleigh-Jeans distribution, which is featured by a marked peak at $k=0$. We recall that complete thermalization to the Rayleigh-Jeans equilibrium spectrum requires extremely long propagation lengths that are not accessible experimentally, as revealed by numerical simulations (the thermalization length is of the order of $10^{5} z_{N L}$ in the example of Fig. 4), or through the analysis of non-equilibrium kinetic equations [17]. At variance with this fully developed equilibrium condensation, here we identify a non-equilibrium precondensation effect that is characterized by a fast relaxation of $n_{0} / I_{0}$ for small propagation lengths available experimentally. Surprisingly, this initial stage of precondensation, featured by an accumulation of relatively longwavelength modes around $k \approx 0$, provides a good indication for the final condensate fraction at Rayleigh-Jeans thermal equilibrium.

We report in Fig. 3(a) the precondensate fraction extracted by fitting the probability distribution (2) to the experimental intensity histogram, as a function of the effective propagation distance $L / z_{N L}$. One clearly observes a continuous increase of $n_{0} / I_{0}$ for different values of the initial speckle correlation length. The Hamiltonian evolution of the random wave can be understood in a microcanonical statistical description, where the total energy $H$ is the relevant parameter in the absence of an external heat bath governing the temperature for a canonical statistical description. Accordingly, we have studied the transition to precondensation by varying the energy $H$, while keeping constant the "number of parti- 

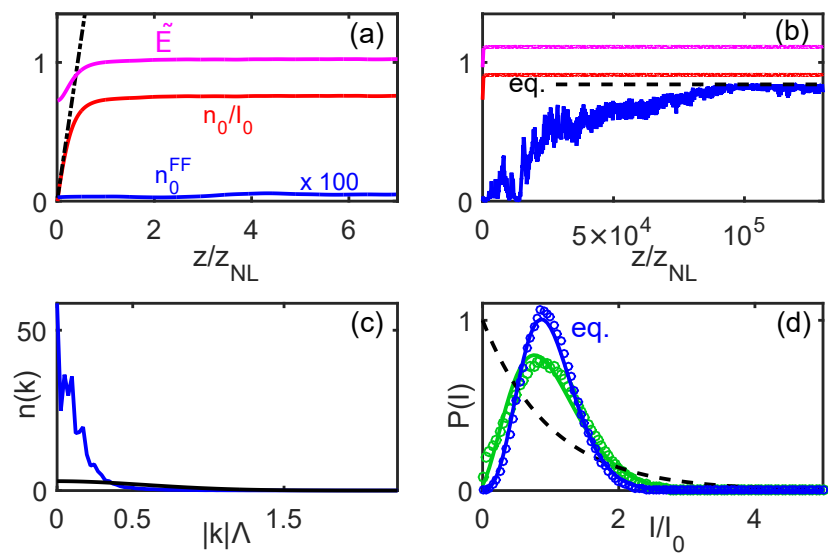

FIG. 4: Numerical simulations of precondensation: Precondensate fraction $n_{0} / I_{0}$ (red lines), zero momentum population $n_{0}^{\mathrm{FF}} / I_{0}$ (blue lines) and normalized kinetic energy $\tilde{E}$ (magenta lines) in early stage (a) and in the long-term evolution (b). The dashed dark lines in (a) denote the analytical prediction for small $z$, in (b) the thermal equilibrium state. (c) Momentum spectrum of the initial wave at $z=0$ (black line) and at $z=100 z_{N L}$ (blue line), showing an accumulation of particles 'near' $k \sim 0$. (d) Near field intensity histogram: initial field (dark dashed line), after $z=100 z_{N L}$ (green circles) and fit of Eq.(2) (green line), and corresponding intensity histograms after $z=1.5 \times 10^{5} z_{N L}$ (in blue).

cles' ( $I_{0}$ fixed). Such a transition is reported in Fig. 3(b) as a function of a dimensionless Hamiltonian, which can be conveniently expressed in terms of the ratio of the healing length $\Lambda$ and the initial correlation length $\sigma_{c}$ : $\tilde{H}=1+\left(\Lambda / \sigma_{c}\right)^{2}[30]$. This representation shows that the nonlinear evolution consists of an exchange between the initial linear contribution $\langle E\rangle_{0} \propto 1 / \sigma_{c}^{2}$, and the initial nonlinear contribution $\langle U\rangle_{0} \propto \gamma I_{0}^{2}$ of the Hamiltonian. This total energy can be tuned in our experiment by either changing the correlation length $\sigma_{c}$, or the nonlinear index of refraction (by changing the laser-atom detuning or the laser intensity). As a remarkable result, the precondensate fraction seems to only depend on the ratio between the linear and nonlinear contributions, $\langle E\rangle_{0} /\langle U\rangle_{0}=\left(\Lambda / \sigma_{c}\right)^{2}$. We report in Fig. 3(b) $n_{0} / I_{0}$ as a function of $\left(\Lambda / \sigma_{c}\right)^{2}$ for various different initial correlation lengths and different values of the nonlinear interaction. The collapse of the data to an almost unique universal curve is a good indicator of the relevance of $\tilde{H}=1+\left(\Lambda / \sigma_{c}\right)^{2}$ to describe precondensation. Note however that there is some dispersion in the positions of the different curves for small precondensate fractions, a feature that can be ascribed to the impact of the nonlocal nonlinearity $[59,60]$.

Numerical simulations of the NLS Eq.(1) show that the precondensate fraction increases in a significant way in the initial stage (see Fig. 4(a)), a feature that can be described analytically: $\frac{n_{0}}{I_{0}} \simeq 2 \sqrt{2} \frac{\Lambda}{\sigma_{c}} \frac{z}{z_{N L}}$ [30]. Note that, as compared to the theory and simulations, the experimental results show a delay for the initial growth of $n_{0} / I_{0}$ and its subsequent saturation, a feature that can be associated to a first correction of a nonlocal nonlinearity [30]. The growth of $n_{0} / I_{0}$ then rapidly saturates to a quasistationary value after few nonlinear lengths $z_{N L}$. This fast process is characterized by a transfer and subsequent equilibration of the kinetic $(E)$ and nonlinear $(U)$ energies to their prethermalized quasi-steady values, which in turn determine the amount of precondensate fraction $n_{0} / I_{0}$ [30]. In marked contrast to such a short-time relaxation of $E$ and $n_{0}$, the spectrum of the random wave exhibits a very slow thermalization to the Rayleigh-Jeans equilibrium distribution. This is revealed by the far-field zero-momentum condensate $n_{0}^{\mathrm{FF}}=n(k=0)$, whose relaxation toward the thermal equilibrium state requires several thousands of nonlinear propagation lengths, see Fig. 4(b) (blue line). Note that this slow relaxation is set by an analogue of photon-photon collisions: The corresponding collision rate scales as $1 / \gamma^{2}$ [17], in contrast to the nonlinear length $z_{N L}$ that scales as $1 / \gamma$ and which is reminiscent of the chemical potential in Bose-Einstein condensates. The far-from equilibrium nature of the precondensation process is also evidenced by the fact that the system does not exhibit long-range phase order, as it would be expected for the thermalized 2D NLS equation below the Berezinskii-Kosterlitz-Thouless transition $[61,62]$. Indeed, precondensation is characterized by a fast decay of the correlation function [30], which is in contrast with the power-law behavior found at equilibrium. This means that precondensation does not refer to a "quasi-condensate" in the sense of the BerezinskiiKosterlitz-Thouless theory [62].

Precondensation is characterized by an accumulation of particles (power) toward $k \sim 0$, as revealed by the far-field spectrum (momentum distribution) in Fig. 4(c). Note that this strongly nonlinear effect $\left(\langle E\rangle_{0}<\langle U\rangle_{0}\right)$ cannot be described by a weak turbulence kinetic approach $[8,16,20]$. This shows an important property, namely the multimode nature of the effect of precondensation. At variance with $n_{0}^{\mathrm{FF}}$ that refers to the pure zeromomentum occupation, here the precondensate refers to a slowly varying coherent field $\psi_{c}(\boldsymbol{r}, z)$, characterized by low-frequency components $k \lesssim 1 / \Lambda$. The conventional decomposition of the field discussed above through Eq.(2) can then be refined by the substitution $\sqrt{n_{0}} \rightarrow \psi_{c}(\boldsymbol{r}, z)$, i.e., $\psi(\boldsymbol{r}, z)=\psi_{c}(\boldsymbol{r}, z)+\phi(\boldsymbol{r}, z)$, where $\phi(\boldsymbol{r}, z)$ denotes the rapidly varying incoherent component and $n_{0}=\left\langle\left|\psi_{c}\right|^{2}\right\rangle$. Importantly, the multimode analysis reveals that the bare intensity distribution (2) is well corroborated by a refined multimode intensity distribution, as revealed by the remarkable agreement between the simulations and the bare intensity distribution (2), see Fig. 4(d) at $z=100 z_{N L}$ and at full equilibrium $\left(z=150 \times 10^{3} z_{N L}\right)$. In addition, precondensation proves robust with respect to the intensity moments $\left(\left\langle I^{p}\right\rangle\right)$ used to compute $n_{0} / I_{0}$, 
or the frequency cut-off that is known to regularize the ultraviolet catastrophe inherent to classical waves. These aspects validate the simple model (2) used to analyze precondensation [30].

In conclusion, we have reported the observation of a phenomenon of non-equilibrium precondensation of classical waves in two dimensions. This experiment can be extended to study a classical analogue of Bose-Einstein condensation after a sudden quench below the critical temperature (in 3D) [48, 49], the Berezinskii-KosterlitzThouless transition (in 2D) [61], or the formation of nonthermal fixed points $[63,64]$. The possibility of shaping the initial conditions further allows the study of the growth of long range coherence using, e.g., a Gaussian beam with small fluctuations corresponding to nonlinear filtering of high frequency components [65], in relation with spatial beam self-cleaning in multimode fibers [66, 67]. This experimental platform also paves the way to the study of a variety of phenomena in the key area of quantum fluids of light, such as superfluid behaviors [3, 68-71], strongly nonlinear shocks [72, 73], nonlocal effects $[59,60]$, the development of turbulence cascades $[8,9,74]$, or quench dynamics in the framework of the Kibble-Zurek mechanism [75].

[1] S. Pitaevskii, L. Stringari, Bose-Einstein Condensation (Oxford Science Publications, 2003).

[2] J. Kasprzak et al., Bose-Einstein condensation of exciton polaritons, Nature (London) 443, 409 (2006).

[3] I. Carusotto, C. Ciuti, Quantum fluids of light, Rev. Modern Phys. 85 299-366 (2013).

[4] T. Byrnes, N. Young Kim, Y. Yamamoto, Excitonpolariton condensates, Nature Phys. 10, 803 (2014).

[5] J. Klaers, J. Schmitt, F. Vewinger, M. Weitz, BoseEinstein condensation of photons in an optical microcavity, Nature 468, 545 (2010).

[6] S. Dyachenko, A.C. Newell, A. Pushkarev, V.E. Zakharov, Optical turbulence: Weak turbulence, condensates and collapsing filaments in the nonlinear Schrödinger equation. Physica D 57, 96-160 (1992).

[7] A.C. Newell, S. Nazarenko, L. Biven, Wave turbulence and intermittency, Physica D 152, 520 (2001).

[8] S. Nazarenko, Wave Turbulence (Springer, Lectures Notes in Physics, 2011).

[9] A.C. Newell, B. Rumpf, Wave Turbulence, Annu. Rev. Fluid Mech. 43, 59-78 (2011).

[10] C. Connaughton, C. Josserand, A. Picozzi, Y. Pomeau, S. Rica, Condensation of Classical Nonlinear Waves, Phys. Rev. Lett. 95, 263901 (2005).

[11] S. Nazarenko, M. Onorato, Wave turbulence and vortices in Bose-Einstein condensation, Physica D 219, 1 (2006).

[12] G. Düring, A. Picozzi, S. Rica, Breakdown of weakturbulence and nonlinear wave condensation, Physica D 238, 1524 (2009).

[13] G. Krstulovic, M. Brachet, Energy cascade with smallscale thermalization, counterflow metastability, and anomalous velocity of vortex rings in Fourier-truncated
Gross-Pitaevskii equation G. Krstulovic, M. Brachet, Phys. Rev. E 83, 066311 (2011).

[14] C. Sun, S. Jia, C. Barsi, S. Rica, A. Picozzi, J. Fleischer, Observation of the kinetic condensation of classical waves, Nature Phys. 8, 471-473 (2012).

[15] A. Rückriegel, P. Kopietz, Rayleigh-Jeans Condensation of Pumped Magnons in Thin-Film Ferromagnets, Phys. Rev. Lett. 115, 157203 (2015).

[16] V.E. Zakharov, V.S. L'vov, G. Falkovich, Kolmogorov Spectra of Turbulence I (Springer, Berlin, 1992).

[17] A. Chiocchetta, P.E. Larré, I. Carusotto, Thermalization and Bose-Einstein condensation of quantum light in bulk nonlinear media, Europhys. Lett. 115, 24002 (2016).

[18] A. Picozzi, J. Garnier, T. Hansson, P. Suret, S. Randoux, G. Millot, D. Christodoulides, Optical wave turbulence: Toward a unified nonequilibrium thermodynamic formulation of statistical nonlinear optics, Phys. Reports 542, 1-132 (2014).

[19] J. Laurie, U. Bortolozzo, S. Nazarenko, S. Residori, Onedimensional optical wave turbulence: experiment and theory. Phys. Rep. 514, 121-175 (2012).

[20] A. Dyachenko, G. Falkovich Condensate turbulence in two dimensions Phys. Rev. E 54, 5095 (1996).

[21] J. Berges, S. Borsanyi, C. Wetterich, Prethermalization, Phys. Rev. Lett. 93, 142002 (2004).

[22] B. Bertini, F. H. L. Essler, S. Groha, N. J. Robinson, Prethermalization and Thermalization in Models with Weak Integrability Breaking, Phys. Rev. Lett. 115, 180601 (2015)

[23] P.-E. Larré, I. Carusotto, Prethermalization in a quenched one-dimensional quantum fluid of light, Eur. Phys. J. D 70: 45 (2016).

[24] T. Langen, T. Gasenzer, J. Schmiedmayer, Prethermalization and universal dynamics in near-integrable quantum systems, Journal of Statistical Mechanics: Theory and Experiment 6, 064009 (2016).

[25] A. Campa, T. Dauxois, S. Ruffo, Statistical mechanics and dynamics of solvable models with long-range interactions, Physics Reports, 480, 57 (2009).

[26] S. Schütz, G. Morigi Prethermalization of Atoms Due to Photon-Mediated Long-Range Interactions, Phys. Rev. Lett. 113, 203002 (2014).

[27] M. Gring, M. Kuhnert, T. Langen, T. Kitagawa, B. Rauer, M. Schreitl, I. Mazets, D. Adu Smith, E. Demler, J. Schmiedmayer, Relaxation and Prethermalization in an Isolated Quantum System, Science, 337, 1318 (2012).

[28] S. Trotzky, Y-A. Chen, A. Flesch, I. P. McCulloch, U. Schollwöck, J. Eisert, I. Bloch, Probing the relaxation towards equilibrium in an isolated strongly correlated onedimensional Bose gas, Nature Physics 8, 325 (2012).

[29] T. Langen, S. Erne, R. Geiger, B. Rauer, T. Schweigler, M. Kuhnert, W. Rohringer, I.E. Mazets, T. Gasenzer, J. Schmiedmayer, Experimental observation of a generalized Gibbs ensemble, Science 348, 207-211 (2015).

[30] See Supplemental Material [url] for a detailed description of the experimental methods (calibration of nonlinearity, correlation length of the incindent speckle beam) and of the theoretical developments (NLS model, robustness of precondensation, nonlocal nonlinearity, $1 \mathrm{D}$ vs $2 \mathrm{D}$ comparison, moment analysis of precondensation), which includes Refs. [31-41].

[31] Y. Wang, M. Saffman, Experimental study of nonlinear focusing in a magneto-optical trap using a Z-scan technique, Phys. Rev. A 70, 013801 (2004). 
[32] Y. R. Shen, The Principles of Nonlinear Optics, New York: J. Wiley (1984)

[33] F. Simoni, Nonlinear Optical Properties of Liquid Crystals and Polymer Dispersed Liquid Crystals, World Scientific (1997).

[34] G.P. Agrawal, Nonlinear Fiber Optics, Academic Press (2013).

[35] L. Deng, K. He, T. Zhou and C. Li, Formation and evolution of far-field diffraction patterns of divergent and convergent Gaussian beams passing through self-focusing and self-defocusing media J. Opt. A: Pure Appl. Opt. 7, 409-415 (2005)

[36] E. V. Garcia Ramirez, M. L. Arroyo Carrasco, M. M. Mendez Otero, S. Chavez Cerda, and M. D. Iturbe Castillo, Far field intensity distributions due to spatial self phase modulation of a Gaussian beam by a thin nonlocal nonlinear media Opt. Express 18, 22067-22079 (2010)

[37] J. Berges, T. Gasenzer, Quantum versus classical statistical dynamics of an ultracold Bose gas, Phys. Rev. A 76, 033604 (2007)

[38] P. Walczak, S. Randoux, and P. Suret, Optical Rogue Waves in Integrable Turbulence, PRL 114, 143903 (2015).

[39] J.M. Soto-Crespo, N. Devine, N. Akhmediev, Integrable Turbulence and Rogue Waves: Breathers or Solitons?, Phys. Rev. Lett. 116, 103901 (2016).

[40] M. Abramowitz, I. Stegun, Handbook of mathematical functions, Dover Publications, New-York, 1965 (sec. 13.1).

[41] A. Picozzi, S. Rica, Condensation of classical optical waves beyond the cubic nonlinear Schrödinger equation, Opt. Commun. 285, 5440 (2012).

[42] C. Conti, M. Leonetti, A. Fratalocchi, L. Angelani, G. Ruocco, Condensation in disordered lasers: theory, 3D simulations and experiments. Phys. Rev. Lett. 101, 143901 (2008).

[43] R. Weill, B. Fischer, O. Gat, Light-mode condensation in actively-mode-locked lasers, Phys. Rev. Lett. 104, 173901 (2010).

[44] G. Oren, A. Bekker, B. Fischer, Classical condensation of light pulses in a loss trap in a laser cavity, Optica 1, 145 (2014).

[45] E. Turitsyna, G. Falkovich, A. El-Taher, X. Shu, P. Harper, S. Turitsyn, Optical turbulence and spectral condensate in long fibre lasers, Proc. R. Soc. A 468, 2145 (2012).

[46] E. Turitsyna, S. Smirnov, S. Sugavanam, N. Tarasov, X. Shu, S. Babin, E. Podivilov, D. Churkin, G. Falkovich, S. Turitsyn, The laminar-turbulent transition in a fibre laser, Nature Photon. 7, 783 (2013).

[47] M. Köhl, M. J. Davis, C. W. Gardiner, T. W. Hänsch, T. Esslinger, Growth of Bose-Einstein Condensates from Thermal Vapor, Phys. Rev. Lett. 88, 080402 (2002).

[48] I. Shvarchuck, Ch. Buggle, D.S. Petrov, K. Dieckmann, M. Zielonkowski, M. Kemmann, T.G. Tiecke, W. von Klitzing, G.V. Shlyapnikov, J.T.M. Walraven, BoseEinstein Condensation into Nonequilibrium States Studied by Condensate Focusing, Phys. Rev. Lett. 89, 270404 (2002).

[49] M. Hugbart, J. A. Retter, A. F. Varon, P. Bouyer, A. Aspect, M.J. Davis, Population and phase coherence during the growth of an elongated Bose-Einstein condensate, Phys. Rev. A 75, 011602(R) (2007).
[50] R.P. Smith, S. Beattie, S. Moulder, R.L.D. Campbell, Z. Hadzibabic, Condensation Dynamics in a QuantumQuenched Bose Gas, Phys. Rev. Lett. 109, 105301 (2012).

[51] P. Suret, S. Randoux, Far field measurement in the focal plane of a lens: a cautionary note, arXiv:1307.5034 (2013).

[52] Y. Bromberg, Y. Lahini, E.Smalland, Y. Silberberg, Hanbury Brown and Twiss interferometry with interacting photons, Nature Photonics 4, 721 (2000).

[53] Y. Silberberg, Y. Lahini, Y. Bromberg, E. Small, R. Morandotti, Universal Correlations in a Nonlinear Periodic 1D System, Phys. Rev. Lett. 102, 233904 (2009).

[54] S. Randoux, P. Walczak, M. Onorato, P. Suret, Nonlinear random optical waves: Integrable turbulence, rogue waves and intermittency, Physica D 333, 323 (2016).

[55] M. Onorato, D. Proment, G. El, S. Randoux, P. Suret, On the origin of heavy-tail statistics in equations of the Nonlinear Schrödinger type, Phys. Lett. A 380, 3173 (2016).

[56] D. S. Petrov, M. Holzmann, G. V. Shlyapnikov, BoseEinstein Condensation in Quasi-2D Trapped Gases, Phys. Rev. Lett. 84, 2551 (2000).

[57] T. Yefsah, R. Desbuquois, L. Chomaz, K.J. Günter, J. Dalibard, Exploring the Thermodynamics of a TwoDimensional Bose Gas, Phys. Rev. Lett. 107, 130401 (2011).

[58] P.-E. Larré, I. Carusotto, Propagation of a quantum fluid of light in a cavityless nonlinear optical medium: General theory and response to quantum quenches, Phys. Rev. A 92, 043802 (2015).

[59] S. Skupin, M. Saffman,W. Krolikowski, Nonlocal Stabilization of Nonlinear Beams in a Self-Focusing Atomic Vapor. Phys. Rev. Lett. 98, 263902 (2007).

[60] D. Vocke, T. Roger, F. Marino, E. M. Wright, I. Carusotto, M. Clerici, D. Faccio, Experimental characterization of nonlocal photon fluids. Optica 2, 484 (2015).

[61] Z. Hadzibabic, P. Krüger, M. Cheneau, B. Battelier, J. Dalibard, Berezinskii-Kosterlitz-Thouless crossover in a trapped atomic gas, Nature 441, 1118 (2006).

[62] S. Nazarenko, M. Onorato, and D. Proment, BoseEinstein condensation and Berezinskii-KosterlitzThouless transition in the two-dimensional nonlinear Schrödinger model, Phys. Rev. A 90, 013624 (2014).

[63] B. Nowak, S. Erne, M. Karl, J. Schole, D. Sexty, T. Gasenzer, Non-thermal fixed points: universality, topology \& turbulence in Bose gases, in Strongly Interacting Quantum Systems out of Equilibrium, edited by T. Giamarchi, A. Millis, O. Parcollet, H. Saleur, L. Cugliandolo, Lecture Notes of the Les Houches Summer School (Oxford University Press, 2016).

[64] J. Berges, B. Wallisch, Nonthermal fixed points in quantum field theory beyond the weak-coupling limit, Phys. Rev. D 95, 036016 (2017).

[65] G. Labeyrie, T. Ackemann, B. Klappauf, M. Pesch, G.L. Lippi, R. Kaiser, Nonlinear beam shaping by a cloud of cold Rb atoms, Eur. Phys. J. D 22, 473 (2003)

[66] L.G. Wright, Z. Liu, D.A. Nolan, M.-J. Li, D. N. Christodoulides, F.W. Wise, Self-organized instability in graded-index multimode fibres, Nature Photon. 10, 771 (2016).

[67] K. Krupa, A. Tonello, B.M. Shalaby, M. Fabert, A. Barthélémy, G. Millot, S. Wabnitz, V. Couderc, Spatial beam self-cleaning in multimode fibres, Nature Photon. 
11, 237 (2017)

[68] A. Amo, S. Pigeon, D. Sanvitto, V. G. Sala, R. Hivet, I. Carusotto, F. Pisanello, G. Leménager, R. Houdré, E. Giacobino, C. Ciuti, A. Bramati, Polariton Superfluids Reveal Quantum Hydrodynamic Solitons, Science 332, 1167 (2011).

[69] D. Sanvitto, S. Pigeon, A. Amo, D. Ballarini, M. De Giorgi, I. Carusotto, R. Hivet, F. Pisanello, V. G. Sala, P. S. S. Guimaraes, R. Houdré, E. Giacobino, C. Ciuti, A. Bramati, G. Gigli, All-optical control of the quantum flow of a polariton condensate, Nat. Photonics 5, 610 (2011).

[70] G. Nardin, G. Grosso, Y. Léger, B. Pietka, F. MorierGenoud, B. Deveaud-Plédran, Hydrodynamic nucleation of quantized vortex pairs in a polariton quantum fluid, Nature Phys. 7, 635 (2011).

[71] D. Vocke, K. Wilson, F. Marino, I. Carusotto, E.M.
Wright, T. Roger, B.P. Anderson, P. Öhberg, D. Faccio, Role of geometry in the superfluid flow of nonlocal photon fluids, Phys. Rev. A 94, 013849 (2016).

[72] M. Onorato, S. Residori, F. Baronio, editors Rogue and shock waves in nonlinear dispersive media, (Springer, Lectures Notes in Physics, 2016).

[73] G. Xu, D. Vocke, D. Faccio, J. Garnier, T. Rogers, S. Trillo, A. Picozzi, From coherent shocklets to giant collective incoherent shock waves in nonlocal turbulent flows, Nature Comm. 6, 8131 (2015).

[74] N. Navon, A.L. Gaunt, R.P. Smith, Z. Hadzibabic, Emergence of a turbulent cascade in a quantum gas, Nature 539, 72-75 (2016).

[75] W. Zurek, Cosmological Experiments in Superfluid Helium?, Nature 317, 505 (1985). 